second group possessed higher values of all parameters in comparison to those from the first and the third groups.

Lesser development of bone tissue revealed by a decreased arm length and size of the major joints probably reflect the anti-androgenic effect of OCPs before and during puberty in males of the first group, while the opposite trend revealed in females from the second group may result from estrogenic effect of mild doses of OCPs. Differences in body mass, waist perimeter and skinfold thickness possessed by volunteers from regions with different environmental conditions may reflect the previously described influence of endocrine disruptor chemicals, such as OCPs, on the balance of leptin, whose functions are established in puberty.

\section{FEVER PHOBIA IN CROATIAN FEVER PHOBIA IN CROATIAN PARENTS - WHAT DO THEY KNOW, BELIEVE AND DO WHEN DEALING WITH A FEBTILE CHILD?}

Borna Biljan*, Sanja Dorner. University of J. J. Strossmayer, Faculty of Medicine; Clinical hospital centre Osijek, Department of pe

\subsection{6/archdischild-2021-europaediatrics.28}

The primary goal of this research was to get some insight into knowledge, beliefs and practices of Croatian parents of febrile children. Are they prone to spontaneous reactions caused by an exaggerated fear of fever? To investigate the extent of their 'fever phobia', there was a need to ascertain the duration and the height of child's fever at which parents decided to consult their paediatrician, as well as what possible complications parents associated with high fever. The aim of this study was also to compare beliefs, practices and the sources of information concerning fever management according to the level of the parents' education and the number of their children.

Parents of 64 febrile children visiting primary paediatrician were interviewed using an anonymous questionnaire with fever-related questions, comprising fever management.

Most parents, $70 \%$ of them, claim that high fever is not harmful if treated properly. Children were taken to a paediatrician with fever of $39.1^{\circ} \mathrm{C}$ by $45 \%$ of parents. Although the aforementioned temperature is deemed dangerous by as much as $67 \%$ of parents questioned, the less educated parents were more prone to visit the paediatrician even at lower temperatures. Even more worryingly, 33\% of all questioned parents came to the paediatrician in under 24 hours of fever's onset. It should be noted that some of the more educated parents listed more severe possible complications such as brain damage and death of the child; while on the other hand, lowering of the child's immunity worried more the less educated parents. Even the sources of information on fever management, apart from paediatricians, varied among parents, with educated parents additionally consulting internet sources in contrast to less educated parents who preferred the pharmacist's advice. No significant difference in parental fever management was observed according to the number of children in the household.

It seems that the proper treatment of fever with children is still insufficiently known to most Croatian parents. The 'fever phobia' could cause dilemmas regardless of the level of education of parents who then turn to their health providers for additional advice. The later might deepen the confusion, due to the lack of uniform guidelines and practice for antipyretic treatment of children, especially preschool ones. Further research in this field could lead to better understanding of the problem and possible creation of Croatia's paediatric guidelines for antipyretic therapy of the febrile child.

\section{WHEN ONLY BIOPSY CAN PROVIDE AN ANSWER}

Ana Rita Curval*, Beatriz Vieira, Juliana da Silva Cardoso, Maria José Dinis. Pediatrics Department, UAG da Mulher e da Criança, Centro Hospitalar Universitário de São João, Porto

\subsection{6/archdischild-2021-europaediatrics.29}

Introduction Peripheral lymphadenopathy in children is generally benign and self-limited. However, it can be a manifestation of serious underlying disease, so differential diagnosis is essential.

Case Report We report a case of a 4-year-old male, previously healthy, presented to the paediatric emergency department with a painless right cervical tumefaction, with about one month of evolution. Fever and weight loss were denied, as well as previous illness and contact with cats. Two antibiotic therapy cycles were performed previously, due to likely bacterial infection, without showing clinical improvement.

On physical examination: Good overall appearance. Rosy faced and hydrated.

Right cervical tumefaction $(5 * 3 \mathrm{~cm}$, painless to palpation, without local inflammatory signs). Without other palpable lymphadenopathies or hepatosplenomegaly.

Laboratory tests revealed: hemoglobin 11,8g/dL; neutrophil series count $8,74 \times 109 / \mathrm{L}$; platelet count 376 x109/L; no changes in kidney and liver function; C-reactive protein and Erythrocyte sedimentation rate were negatives.

Due to the persistence of tumefaction, hospitalization was decided for etiological investigation. Serological tests for HIV, CMV, Toxoplasmosis and Bartonella henselae were all negative. Tuberculin skin testing was negative.

Chest radiograph showed no changes. Abdominal ultrasonography with two lymphadenopathies of 11 and $15 \mathrm{~mm}$ in retroperitoneal space, without hepatoesplenomegaly. Cervical ultrasonography revealed a hypoecoggenic nodular image of regular and well-defined contour in the right jugulo-digastric chain, measuring $41 \mathrm{mmx} 16 \mathrm{~mm}$, and showing no adipose hilum, suggesting lymphadenopathy. Aditionally, there were other smaller lymphadenopathies.

Although studies have suggested a benign etiology, due to persistence of tumefaction, a biopsy of the lesion was performed and indicated Burkit Lymphoma.

Conclusion With this work, the authors intend to highlight that if after four weeks of observation and/or empiric therapy, the diagnosis remains uncertain and the lymph node has not regressed in size, a biopsy should be warranted, as only this can confirm or exclude for sure a diagnosis.

\section{A CLINICAL CASE OF A NO EVIDENCE-BASED MEDICAL TREATMENT OF CYTOMEGALOVIRUS INFECTION IN AN INFANT}

Elizaveta Leonova*, Alina Pankova, Anastasia Lamasova, Ekaterina Dubonosova, Anna Alekseeva, Elena Vishneva, Leyla Namazova-Baranova. The Research Institute of Pediatrics and Child Health of the Central Clinical Hospital of the Russian Academy of Sciences of the Ministry of Science and Higher Education of the Russian Federation

10.1136/archdischild-2021-europaediatrics.30 
Background Cytomegalovirus, which belongs to the herpes viruses group, is the most frequent cause of congenital infection. The fetus may be silently infected in utero, as a result of initial infection or reactivation of a chronic infection in the mother. This may lead to different failures of the child's organs and systems. The presence of antibodies to cytomegalovirus (CMV) in infants, even without clinical signs of infection, often leads to unreasonable medical treatment.

Patients and Methods The parents of the 2-month-old child appealed to the department to verify the correctness of prescribed treatment. A high level of CMV IgG was detected in maternal blood after pregnancy. That's why congenital CMV infection was suspected in 1-month -old infant. His laboratory tests revealed high level of CMV IgG, but CMV DNA wasn't found by PCR in blood, urine, saliva. In spite of this, the baby was diagnosed with congenital CMV infection and was treated with anti-human anticytomegalovirus immunoglobulin (2 doses). However, antibodies titer was at the same level on repeat testing. On physical examination in our department: the condition of the child was satisfactory, cognitive development was normal.

Results Diagnosis of congenital CMV infection isn't correct, according to negative CMV DNA PCR in blood, saliva, urine and lack of clinical manifestation (microcephaly, jaundice, petechial rash, hepatosplenomegaly, hepatitis, pneumonitis, sensorineural hearing loss, etc.). Therefore, further examination and specific immunoglobulin therapy aren't needed, dynamic observation is recommended. The child's condition remains satisfactory at the age of 4 months, there aren't any complaints from his parents.

Conclusion The main diagnostic test of congenital CMV infection is PCR of body fluids, which means that serological research should not be used in routine diagnostics. The detection of CMV IgG in clinically healthy infants isn't a criteria for this diagnose and does not require specific treatment.

\section{DON'T FORGET ABOUT RISK OF PERTUSSIS IN CHILDREN WITH ASTHMA}

Alina Pankova*, Anastasia Lamasova, Ekaterina Dubonosova, Elizaveta Leonova, Vera Kalugina, Anna Alexeeva, Marina Fedoseenko, Leila Namazova-Baranova. Research Institute of Pediatrics and Child Health CCH RAS, Ministry of Science and Higher Education

\subsection{6/archdischild-2021-europaediatrics.31}

Background Pertussis, also known as 'whooping cough,' is a highly contagious respiratory disease, caused by Bordetella pertussis.

Nowadays it primarily affects children too young to have completed the full course of vaccinations, children with chronic lung diseases, teenagers and adults whose immunity has faded. Incidence of pertussis among children 7-14 years old in Russia increased twice in 2019, compared with 2018, possibly due to fading immunity among vaccinated children. However, there is still no pertussis booster vaccination for children over 6 years of age in the Russian National Immunization Schedule.

Case Report A 10-year-old boy, appealed to the department with complaints of paroxysmal nonproductive cough, becoming nocturnal for the last 14 days, without fever. The boy was diagnosed with asthma at age of 7 years, for the last 1,5 years he had asthma remission and hadn't received controller therapy. The boy was vaccinated according to the National
Immunization Schedule of the Russian Federation. At the previous pediatrician examination there wasn't any additional sounds, normal breath during the lungs' auscultation. However, the doctor decided that it was asthma exacerbation caused by a viral disease. Boy was treated with inhaled salmeterol/fluticasone propionate combination without any significant effect: the cough became worse, hacking, nocturnal with "whooping» sounds.

On physical examination difficulty breathing through the nose was detected.

On auscultation, normal breath without any additional sounds were audible over both lungs. The remainder of the physical examination was unremarkable.

Epidemiological anamnesis: The mother of the child had frequent coughing paroxysms for the last 2 weeks.

Results Pertussis was suspected because of the long-term coughing paroxysms, lack of response to bronchial asthma controller therapy, positive epidemiological anamnesis. Bordetella pertussis DNA was detected by PCR in nasopharynx mucus. Also, positive IgM (12.6 IU/mL) and $\operatorname{IgG}(60.8 \mathrm{IU} / \mathrm{mL})$ antibodies to B. pertussis were found in the blood serum. The boy was treated with clarithromycin. Since the beginning of treatment there was significant improvement in health: the cough became rare without whooping.

Conclusion Children with bronchial asthma and lack of response to controller therapy ought to be suspected with whooping cough. Particular attention should be given to schoolchildren and adolescents, especially with chronic lung diseases: vaccination against pertussis at the age of 6 and 1213 years is recommended, possibly due to fading immunity among vaccinated children over 6 years of age.

\section{CLINICAL CASE OF HYPOHYDROTIC ECTODERM DYSPLASIA: SPECIFIC SYMPTOMS FROM THE ENT ORGANS}

S Gubanova*, I Zelenkova, L Namazova-Baranova, A Alekseeva, L Aslamazyan. The Research Institute of Pediatrics and Child Health of the Central Clinical Hospital of the Russian Academy of Sciences of the Ministry of Science and Higher Education of the Russian Federation

\subsection{6/archdischild-2021-europaediatrics.32}

Ectoderm dysplasia is a rare hereditary disease resulting from mutations in genes encoding the development of ectoderm (mainly ectodysplazin-A receptor genes). The most common form is X-linked hypohydrotic ectoderm dysplasia. The prevalence of this form of the disease is estimated from 1.6 to 22 cases per 100,000 newborns. Clinical symptoms are diverse and can be manifested by impaired function of various systems and organs.

Methods Boy X. 1y $6 \mathrm{~m}$ old, vaccinated only against BCG at birth, saw the ENT doctor with complaints of recurring purulent rhinitis with the formation of crusts in the nasal cavity with a fetid odor. Local therapy (decongestants, elimination therapy) had no effect. Examination by an otorhinolaryngologist revealed chronic atrophic rhinitis. Forming nasal septum perforation and epithelial plugs in the external auditory canals were detected, as well as conical teeth.

Local therapy of rhinitis was prescribed with medications containing D-panthenol (for moisturizing the mucous membrane and prevention nasal septum perforation) and an 